\title{
DESIGUALDAD Y MOVIMIENTOS ALTERNATIVOS EN EL CAPITALISMO ACTUAL: UN CONTEXTO PARA EL 15-M
}

\author{
Inequality and some alternative movements in today capitalism: \\ a context for the 15-M
}

JosÉ MARÍA TORTOSA BLASCO ${ }^{1}$

\section{Resumen}

El actual sistema mundial se basa en la desigualdad entre territorios y entre grupos sociales (clase, género, raza, cultura) y ha producido históricamente, en dichos grupos, descontentos con sus resultados: rebeldes, «indignados», «protesters» $y$, aunque no frecuentemente, revolucionarios. Las circunstancias contemporáneas de inseguridad económica, energética, alimentaria, medioambiental e incluso física dan una particular coloración a los movimientos alternativos aparecidos en dichas circunstancias a pesar de sus semejanzas con movimientos aparecidos en tiempos anteriores. En el texto se lleva a cabo una revisión de algunos movimientos recientes (piqueteros argentinos, forajidos ecuatorianos, islandeses, "primaveras árabes», revueltas en Grecia, estudiantes chilenos -y otros latinoamericanos-, «indignados» en Israel, alborotadores en Tottenham y «marchistas» del TIPNIS boliviano) para extraer las correspondientes lecciones de cara, sobre todo, al conjunto 15-M, «Occupy Wall Street», 15-O y sus intentos de mundialización.

Palabras clave: sistema mundial, desigualdad, inseguridad, movimientos alternativos, cambio social

\begin{abstract}
The current world system is based on inequality between regions and between social groups (class, gender, race, culture) and has historically produced from these groups, movements of people unhappy with their results: rebels, «indignados», «protesters» and, although infrequently, revolutionaries. The contemporary circumstances of insecurity in the fields of economy, energy, food, environment and even in the physical sense, has created a particular type of alternative movement produced by such circumstances despite their similarities with movements that have appeared in earlier times. In this text a review of some of these recent movements is made (the Argentinean «piqueteros», Ecuadorian «forajidos», Icelanders, «Arab Spring», riots in Greece, Chilean and other Latin-American students, «indignados» in Israel, trouble makers in Tottenham and «marchistas» of the Bolivian TIPNIS) to draw some lessons, above all, from the groups 15-M, «Occupy Wall Street», 15-O and their attempts to become global movements.
\end{abstract}

Key words: world system, inequality, insecurity, alternative movements, social change

Recibido: 28/12/2011 Revisado: 30/04/2012 Aceptado: 08/05/2012 $\quad$ Publicado: 02/11/2012

1. José María Tortosa Blasco. Instituto Interuniversitario de Desarrollo Social y Paz. Universidad de Alicante. Manolo Morán, 33. 03550 San Juan de Alicante. 965655597. jmtortosa3@gmail.com 
Hay momentos en la historia en los que gentes de todo el mundo se alzan para clamar que algo anda mal y que hace falta un cambio. Fue así en los tumultuosos años 1848 y 1968. También lo fue, ciertamente, en 2011. En muchos países hubo rabia y descontento por el desempleo, la distribución de la renta y la desigualdad y se sintió que el sistema es injusto e incluso que está en quiebra.

Joseph Stiglitz (2012) The 99 Percent Wakes Up, The Daily Beast, 3 de mayo.

Las calles se convierten en laboratorios mágicos en los que se crean ciudadanos y camaradas y las ideas radicales adquieren un repentino poder telúrico. Pero ipersistirá este nuevo cometa de protesta en el cielo invernal o es sólo una breve y deslumbrante lluvia de meteoritos?

Mike Davis (2011) Spring Confronts Winter, New Left Review, $\mathrm{N}^{\mathrm{o}} 72$, noviembre-diciembre.

La oleada de heterogéneos «indignados» manifestándose en plazas, bulevares o parques como Tahrir, Sol, Sintagma, Rotschild, San Pablo o Zuccotti (Varios Autores, 2011a; Varios Autores 2011b; Stiglitz, 2012, Chomsky, 2012; Kempf, 2012) tiene precursores. Se puede pensar, como antecedente, en la crisis cíclica de 1929 con la que, en efecto, tiene resonancias. La crisis económica, el descrédito de la democracia, el auge de los movimientos totalitarios cargados de verdades absolutas e intolerantes de cualquier duda y una potencia hegemónica en crisis son elementos a tener ahora en cuenta en la comparación porque son comunes. Pero no se trata de las mismas circunstancias: en el 29 no había conciencia de la huella ecológica ni se discutía sobre el «pico del petróleo». Ni había 1.000 millones de hambrientos en el mundo. En este sentido, la situación actual, al decir de algunos observadores (Chomsky, 2011), no tendría precedentes.

Lo que se va a proponer tiene cuatro partes: 1. la generación de desigualdades como telón de fondo de algunos movimientos sociales; 2. el papel de las nuevas inseguridades como precipitante de las actuales rebeliones y movimientos; 3. enumeración de algunos de estos, como contexto inmediato del 15-M; y 4. los retos que se plantean al 15-M de cara al futuro.

\section{Un sistema que genera y se nutre de desigualdades}

De Rosa Luxemburg a Zygmunt Bauman se ha expuesto que el capitalismo realmente existente funciona mediante la «colonización» de «territorios vírgenes» para la explotación. Esta colonización puede ser física 
(«descubrimientos», conquista, ocupación), puede ser comercial (el neocolonialismo inaugurado por los Estados Unidos al sustituir a Inglaterra como potencia hegemónica colonial) o puede ser financiera. Por otro lado, el sistema actual se habría mantenido pasando del capitalismo del que hablaba Max Weber (austero, ahorrador, calculador, inversor) al capitalismo de consumo y, de éste, al capitalismo del crédito que es el que habría entrado en crisis como todos los modos anteriores de mantener en vigor la norma básica del sistema, que es la del beneficio sin límites físicos, sociales y económicos o, si se prefiere, la de la acumulación incesante de capital. Lo que ahora mantiene vivo al sistema es la colonización del futuro: el beneficio de hoy se obtiene cargando sobre las espaldas no de los «salvajes», de los «primitivos pre-capitalistas», de «los de abajo» en general, sino sobre las generaciones futuras. Eso es, al fin y al cabo, la actual crisis de la deuda.

En este sistema, dado que funciona mediante la desigualdad, se produce lo que en algún momento fue visto como «sus propios sepultureros», es decir, los proletarios, como se afirmaba en el Manifiesto Comunista, aunque los previstos por Marx y Engels no lo consiguieron. Tampoco lo habían conseguido los indignados por los efectos del maquinismo, llamados luditas por la localidad inglesa (Ludd) en la que comenzaron las protestas por la introducción de lo que ahora se llamarían «nuevas tecnologías».

En general, en el sistema se producen reacciones contra la desigualdad tanto interna como externa: clases, «etnias», religiones, género por un lado y colonias y satélites por otro. Algunas de estas desigualdades son consideradas injustas por los perdedores, aunque no por todos ellos, y generan reacciones a favor de estos que son los que pierden en el intercambio colonial que incluye el «colonialismo interior» no solo espacial sino, sobre todo, social. "Los de abajo», o el «99 \%» que es terminología contemporánea, de vez en cuando hacen oír su voz reivindicando derechos, conquistas anteriores mermadas o proyectos alternativos de futuro. No siempre han alcanzado una escala mundial ni siquiera con las sucesivas y heterogéneas Internacionales políticas o sindicales ya que, en contra de lo dicho en el Manifiesto, los proletarios sí que tienen patria: de hecho, el nacionalismo (estatal o subestatal) es la única ideología dominante a escala mundial. Por contra, son frecuentes, por parte de los involucrados en los actuales movimientos alternativos, los pronunciamientos sobre la fuerza que estos están teniendo en sus diversas versiones acompañados por anuncios de una «crisis terminal del sistema».

A pesar de esos anuncios, no es evidente que el capitalismo y su lógica estén llegando a su fin (Streeck, 2011; Williamson, 2012). De momento, hasta ahora las clases dominantes han sido capaces de superar las sucesivas «etapas 
superiores del capitalismo»y los anuncios de «crisis terminales». Primero, mediante la invención del Estado de Bienestar reductor de las contradicciones entre «los de arriba»y «los de abajo». En la actualidad, mediante la aplicación del Estado de Bienestar para ricos, la colonización del futuro y la gestión del miedo.

\section{A la desigualdad se le añaden las inseguridades actuales}

La lógica del sistema, bajo el principio básico del beneficio sin limitaciones y con el efecto de generar descontentos, ha tenido efectos devastadores en la actualidad.

1. Está, antes de todo, la crisis económica que afecta, de momento, a los países centrales en los que un decrecimiento o bajo crecimiento se traduce en reducción de empleo y aumento de problemas que lo acompañan después de que los gobiernos corriesen a salvar a los bancos con problemas y, con ello, entraran en déficit o lo aumentaran. Posteriormente, su necesidad de endeudamiento se fue solventando con préstamos para afrontar los gastos corrientes, no la inversión productiva, reduciendo así el gasto público y, por tanto, introduciendo un nuevo factor de desaceleración.

No es la primera vez que sucede tal cosa (Reinhardt, C.M. y Rogoff, K.S., 2011; Fontana, 2011) aunque probablemente lo sucedido en otras ocasiones ha sido más local o no afectó de la misma forma a los países centrales. Precisamente, a diferencia de la crisis del 29, lo que ahora se produce es una acumulación de problemas nuevos que se añaden, interactuando, a los económicos, generando así una particular inestabilidad del sistema en su conjunto, comenzando por su potencia hegemónica, los Estados Unidos, probablemente en decadencia (Engelhardt, 2011; Smith y Max-Neef, 2011).

2. En primer lugar, están los problemas medioambientales. Además se colonizar el futuro, parece claro que la constante colonización de la Naturaleza llevada a cabo por casi toda la especie humana se ha acelerado alcanzando niveles cuya «huella ecológica» podría llegar a cotas de difícil recuperación homeostática. Es cierto que se puede seguir discutiendo, a veces por motivaciones espurias, si el Planeta se encuentra ante la amenaza (o la realidad) de un cambio climático que es cierto que es rechazado por científicos «negacionistas», por empresas (especialmente algunas petroleras) y por sectores importantes del Partido Republicano estadounidense y sus subordinados. 
Lo que ya no es discutible, sobre todo después del informe de la Universidad de Berkeley ${ }^{2}$, es el hecho del reciente calentamiento global. Sus efectos hasta ahora sobre sequías, inundaciones y malas cosechas han sido suficientemente constatados. Si la especie humana está en riesgo de crisis profunda es pronto para saberlo, pero sí es notable que gobiernos y ciudadanos están dispuestos (y las encuestas internacionales son explícitas al respecto) a sacrificar, a largo plazo, el medioambiente con tal de volver, a corto plazo, a tasas de crecimiento económico que permitan el retorno del empleo.

3. El World Energy Outlook publicado por la Agencia Internacional de la Energía en 2011 reconocía la inminencia (si no presencia) del llamado «pico del petróleo», momento en que el consumo ya se hace a costa de las reservas sin que los nuevos descubrimientos (en Argentina, Brasil o País Vasco por poner ejemplos) sean suficientes para compensar el agotamiento de los recursos aunque aparentemente el consumo siga aumentando.

4. Junto a esta inseguridad energética, aunque hay motivos para desconfiar de algunos planteamientos de organismos internacionales como el Banco Mundial o la FAO, se presenta también una inseguridad alimentaria que, como las anteriores, no afecta por igual a todas las partes del mundo. Si la crisis económica y la energética son problemas sobre todo de los países centrales, los efectos de la crisis medioambiental se notan más, de momento, en los países periféricos y, ciertamente, la crisis alimentaria les afecta igualmente a través de hambrunas. La inseguridad alimentaria tiene múltiples raíces (climáticas, especulativas, energéticas) a las que se añade la concentración de la tenencia de tierras. No es descabellado calcular, consiguientemente, mil millones de subnutridos en el mundo actual, con una pequeña incidencia en los países centrales (en torno a los 19 millones), pero que aparece en proporciones enormes en algunas de las constatables catástrofes humanitarias actuales. No es, entonces, impensable, una hambruna mundial unida a una nueva "crisis del petróleo» (Gorraiz López, 2012)

5. Lo dicho se produce en un ambiente generalizado de descrédito de las democracias realmente existentes y de sus respectivas «clases gobernantes» como las llamaba Bertrand Russell en «The Modern Midas» de 1932, en plena crisis del 29, en términos que podrían ser contemporáneos al decir que «está muy claro que las clases gobernantes del mundo son demasiado ignorantes y estúpidas como para ser capaces de ver más allá del tal problema [el del

2. Disponible en http://berkeleyearth.org/analysis.php 
excesivo poder de las finanzas] y demasiado engreídas como para pedir consejo a quienes podrían ayudarles».

Las encuestas internacionales (también las españolas) dejan a la clase política en puestos bajos en cuanto a su aceptación o la valoración general que provocan en sus respectivos ciudadanos aunque con diferencias de país a país según su historia, cultura política dominante y su progreso o retroceso en la satisfacción de las necesidades básicas de sus ciudadanos ${ }^{3}$. La contradicción que subyace es la de cómo conseguir, sin cambiar las reglas del juego, crecer para crear empleo y, al mismo tiempo, decrecer para responder a los desafíos medioambientales cuando, optando por el primer término, tampoco se consigue el empleo, con lo que los males se acumulan. Y cómo hacerlo desde la política después de una larga etapa en la que el mantra, ahora ya prácticamente en desuso, ha sido «menos Estado, más mercado», cosa que ha producido y acumulado desregulaciones, sobre todo en el sector financiero y ha estado entre los factores que han llevado a la actual crisis económica que, además, se está gestionando en muchos casos sometiendo la política a los dictados de intereses económicos.

6. El resultado de esta acumulación de crisis es el sentimiento de inseguridad que se difunde entre amplias capas de la población. Tal vez no exactamente el «99 \%», pero sí en sectores medios y medio-bajos de los países centrales. Estas inseguridades incluyen, pues, la inseguridad económica: el futuro del propio empleo y de la propia pensión (Estefanía, 2011), pero que solo es una parte del problema. También está la inseguridad energética: los efectos del «pico del petróleo» y la necesaria transición energética para la que no hay muchos mapas. Todo ello no afecta tanto a los países periféricos, que reciben el embate de la inseguridad alimentaria y la medioambiental, sino que afecta, sobre todo, a los países hasta ahora centrales, pero no por ello eternamente centrales y que podrían ser países en vías de subdesarrollo (Therborn, 2007; Lewis, 2012; Newman, 2012).

\section{Reacciones recientes}

Las reacciones ante las desigualdades del sistema han sido frecuentes aunque no han asumido las mismas formas en países centrales que en países

3. Para 2011, los datos internacionales del Pew Research Center están disponibles en http://www.pewglobal.org/database/?indicator=3; los del Latinbarómetro en http:// www.latinobarometro.org/; y el Barómetro de marzo de 2012 del Centro de Investigaciones Sociológicas español en http://www.cis.es/cis/opencm/ES/1_encuestas/estudios/ ver.jsp? estudio $=12684$. 
periféricos. La perspectiva temporal podría iniciarse con la Primavera de los Pueblos en la Europa de 1848 con ramificaciones en una cincuentena de países desafiando a monarcas y autócratas. También hubo reacciones ante la Gran Depresión. Pero parece que es suficiente remontarse a algo más tarde: 1968.

En mayo de 1968, en el cambio de ciclo económico largo de una fase ascendente a una descendente, el movimiento fue mucho más general. Se trataba del «seamos realistas, pidamos lo imposible» y encontraron «la arena debajo de los adoquines» parisinos aunque con ramificaciones en numerosos países no solo centrales, como la ofensiva del Tet en Vietnam, el movimiento contra la guerra en muchas partes del mundo, la Primavera de Praga o la masacre en la plaza de Tlatelolco en México Distrito Federal.

La evaluación de este movimiento no es unánime. Para algunos, como Immanuel Wallerstein, se trató de una «revolución mundial», con causas comunes (el agotamiento de un modelo de Estado) y con efectos perdurables en la percepción de los problemas. Para otros, no tuvo mayores efectos, como pudo verse en los siguientes pasos políticos en Francia (referéndum y elecciones) y en el retorno a la «normalidad».

No son los únicos «indignados» que ha habido en el mundo: un sistema que produce desigualdades tiene que producir también «indignados» con las mismas (Artal, 2011; Lewis, 2012). Se podría incluir el Foro Social Mundial como «movimiento de movimientos» $\mathrm{y}$ «semillero de ideas», aunque tal vez ya no sea el caso (Vivas, 2012) y, sin duda, los neozapatistas del Ejército Zapatista de Liberación Nacional (Aguirre Rojas, 2012). Pero no tiene sentido intentar producir un mapa de escala 1:1. Lo que sigue es una enumeración (necesariamente incompleta) de los movimientos locales recientes con algunas observaciones sobre los mismos que pueden servir de contexto para los movimientos que, como los relacionados con el 15-M, 15-O y los diversos «Occupy», quieren ser mundiales, que todavía son muy heterogéneos y que, por tanto, carecen de una táctica común y de una estrategia común.

\begin{tabular}{|rl|l|}
\hline Movimiento & Observaciones \\
\hline 1. & Piqueteros & Omnipotencia de ideas \\
2. & Forajidos & Evaluación al tiempo \\
3. & Islandeses & Tamaño y tradición \\
4. & Primaveras árabes & Importancia obstáculos \\
5. & Grecia & Frustración y agresividad \\
6. & Estudiantes Chile & Maximalismo \\
7. & Indignados Israel & Lo que no se discute \\
8. & Tottenham & Solo expresión \\
9. & TIPNIS & Instrumentación \\
\hline
\end{tabular}


1. Se traen a colación los piqueteros argentinos (en particular las protestas históricas de 2001-2002 contra la desigualdad en el reparto de las cargas) porque sirve de advertencia fundamental: su eslogan más conocido («Que se vayan todos») fue expresión de un deseo, pero no puso en funcionamiento mecanismos que permitieran su cumplimiento. De hecho, «se quedaron todos» por más que se lograran mayores niveles de «concienciación». Tal vez cambió la «conciencia», pero no mucho más que trascendiese lo local-barrial.

2. En abril de 2005, «forajido» fue el nombre que, en el Ecuador, el presidente Gutiérrez dio a los que se manifestaban contra su presidencia, nombre que fue asumido por los manifestantes que acabaron iniciando la «revolución ciudadana» que daría pie al triunfo de Rafael Correa. La evaluación inmediata que, en el mismo mes de abril, hacía Alberto Acosta (2005), dirigente del que sería el movimiento y después partido de Rafael Correa, concluía diciendo: «Ya se sienten vientos de cambio que serán determinantes para la vida de este pequeño país andino y que, quizás, también sirvan de ejemplo en la región». Tenía razón. Pero el caso ecuatoriano, como el de tantas revoluciones (incluida la Gran Revolución -la francesa-, la Revolución Mexicana y la Revolución de Octubre), muestra que la evaluación no puede hacerse ni en el momento de su inicio ni, mucho menos, en el de su triunfo o apogeo, sino con perspectiva temporal como para saber si ha habido o no una «revolución ciudadana». Destacados ecuatorianos lo niegan ahora.

3. Las "primaveras árabes» son muy heterogéneas (Gutiérrez de Terán, I. y Álvarez-Ossorio, I., 2011) aunque la desigualdad (política, económica, social) aparece de una forma u otra en todas ellas. En efecto, no es lo mismo, por citar a sus comienzos, Túnez que Egipto y, después, no sería lo mismo Bahrein, intervenida por Arabia Saudita, y Libia, intervenida por la OTAN bajo mando estadounidense. O Siria. La advertencia, en este caso, se refiere al conocimiento de los posibles obstáculos que los levantamientos populares pueden encontrar y que, de nuevo, difieren de un lugar a otro y, por tanto, producirán efectos diferentes. En todo caso, revuelta no es revolución y los acontecimientos posteriores han mostrado, además, los problemas que genera el creer que «el cambio está en tu mano» sin percatarse de los efectos que podría tener, por ejemplo, una exaltación de la libertad o de la dignidad, pero con elevados costes para el bienestar o expresiones de identidad a costa de la seguridad. Está por ver si las «primaveras árabes» son seguidas de unos «inviernos árabes» (Álvarez-Ossorio, 2012). De todos modos, sí que cabe resaltar que muchos de los movimientos alternativos recientes, en particular las 
mentadas "primaveras», habrían sido casi imposibles de no ser por las nuevas tecnologías de la información (González-Quijano, 2011; Chouikha, 2011).

4. El caso islandés (Stryker, 2011), ya en 2011, muestra la importancia de la historia reciente (en su caso, de las luchas por la independencia lograda en 1918 como reino y en 1944 como república), de las tradiciones culturales y de lucha y de la población relativamente reducida (algo más de 300.000 habitantes). La onda de protestas que se producen en el mundo, reacción ante el sistema precipitada por las condiciones inmediatas de inseguridad e insatisfacción, se «refracta» en cada circunstancia local de modo que no es tan sencillo exportar o importar métodos y estrategias. Con otra historia, cultura política y condicionamientos es difícil ser «Islandia» con independencia de qué se haya conseguido realmente con las reacciones populares.

5. Las reacciones en Grecia, a los sucesivos «planes de ajuste» y «terapias de choque» impuestas desde el exterior recuerdan lo que, en su momento, se llamaron «IMF riots», las protestas de una población que ve disminuir sus niveles de vida por causa de dichos «planes» o «terapias», disminución que no afecta a todos los ciudadanos de la misma manera, pero que lleva al incremento de suicidios. Tienen elementos en común, en este sentido, con lo sucedido entre los años 70 y 80 en América Latina, pero también los tienen con algunos de los países árabes en los que ha habido «primavera». Hay, sobre todo, emoción y comportamiento expresivo de la misma, que suele ir dirigida contra el gobierno que ha aceptado velis nolis la imposición exterior y no contra el organismo, institución o personas que la han impuesto.

6. El caso de las manifestaciones de los estudiantes chilenos implica también una advertencia: en una negociación, como la que podría haber sido la suya con su gobierno, es aconsejable llegar con peticiones ligeramente superiores a lo que se pretende, para así tener un margen de negociación; si, en cambio, se llega pidiendo exactamente lo que no se está dispuesto a renunciar, el maximalismo de ambas partes puede llevar a un punto muerto. Mucho más problemático sería si, como en mayo del 68 , se entona el «seamos realistas, pidamos lo imposible». De momento, las manifestaciones por una educación de calidad se produjeron, el 24 de noviembre de 2011, en Argentina, Brasil, Chile, Colombia, Costa Rica, Ecuador, Guatemala, Honduras, México, Paraguay, Perú, Uruguay y Venezuela. No es, por tanto, un movimiento de tono menor o puramente local o localista.

7. Los «indignados» de Israel (Badra, 2012) lo son por determinadas condiciones de vida que encuentran inaceptables, en particular las que se refieren 
al acceso a una vivienda digna. Comenzó en julio de 2011 a propósito del precio de determinados alimentos y acabó extendiéndose a plantear la cuestión de la desigualdad social en el país. Conviene, en este caso (y en los demás), levantar acta, además de sus reivindicaciones, también de lo que queda fuera de la agenda de los «indignados», aunque tal silencio no sea más que por no alienarse sectores importantes de la propia sociedad. Porque se puede estar en contra de determinadas políticas de un gobierno sin por ello poner en cuestión otras políticas del mismo gobierno del tipo expansionista con viviendas en los nuevos asentamientos. De hecho, se ha pasado como gato sobre brasas respecto a la situación de Cisjordania y las prácticas israelíes allí.

8. Dos reacciones populares más, ambas muy locales y significativas, pero, al mismo tiempo, muy diferentes entre sí. Por un lado, los disturbios iniciados en Inglaterra, en concreto en Tottenham, agosto de 2011, puramente expresivos del descontento y muy parecidos a los de los «banlieusards» parisinos de 2005: fruto de las inseguridades producidas por una sucesión de recortes sociales y, al mismo tiempo, productoras de nuevas inseguridades en el resto de la población. No había ningún objetivo a alcanzar: solo expresión de rabia y frustración.

9. En el otro extremo, la «larga marcha» en Bolivia de los indígenas contrarios al trazado de una carretera que atravesaría su territorio y parque natural (TIPNIS), sin haberles consultado como pide la Constitución del Estado Plurinacional (Prada, 2011; Gómez Vela, 2012). Objetivo concreto, medios concretos -con mucho esfuerzo y sacrificio- y éxito inmediato al dar marcha atrás el gobierno y la multinacional brasileña que iba a construir la calzada. La advertencia, aquí, es otra: la necesidad de distinguir entre los motivos o las causas de una reacción por un lado y, por otro, las posibilidades de manipulación que pueden proporcionar y que no se convierten en una negación de dichas causas. Fuesen o no fuesen manipulados contra el gobierno del presidente Morales, el hecho es que los indígenas tenían razón, aunque, como en el caso ecuatoriano de los «forajidos», es preciso ver qué sucede en el tiempo y si la «ley corta» abortando la carretera es sucedida, como parece, por prácticas que la favorecen, apoyadas por manifestaciones que pueden haber sido igualmente manipuladas y que han provocado nuevas marchas (Prada, 2012).

10. Se llega, así, al movimiento del 15-M y sus variantes locales y su evolución hacia el 15-O a través de los diversos «Occupy» (Wall Street inicialmente, un 17-S, pero después en más ciudades, por ejemplo inglesas, con la pretensión de «internacionalizarse», del « $99 \% »$ frente al «1\%»). Aparentemente están 
en otra dimensión a pesar de su evidente parentesco con muchos de los anteriores. Joseph Stiglitz (2011) titulaba un artículo con «La globalización de la protesta» en el que se refería a que «El movimiento de protesta que nació en enero en Túnez, para luego extenderse a Egipto y de allí a España, ya es global: la marea de protestas llegó a Wall Street y a diversas ciudades de Estados Unidos» y comentaba «Tenemos un sistema donde a los banqueros se los rescató, y a sus víctimas se les abandonó».

Está por ver si el 15-O se consolida (Wallerstein, 2011). De hecho Zygmund Bauman afirmó en el diario El País (17 de octubre de 2011) que «El 15$\mathrm{M}$ es emocional, le falta pensamiento». Parece un tanto exagerado si se compara con fuentes del mismo movimiento en las que no falta el pensamiento (Varios Autores, 2011b; Varios Autores, 2011c; Domènech, 2011). También está por ver si las indignaciones locales, muchas veces con planteamientos no solo locales sino incluso localistas, se articulan en respuestas colectivas a escala mundial. Uno de los obstáculos que, por lo menos el movimiento Occupy Wall Street, parece ir superando (Doyle, 2011) es la dificultad de reflejar adecuadamente las desigualdades de su propia sociedad en términos de edad, raza y clase social (no solo jóvenes, de raza blanca y de clase media). Lo que sí se pueden plantear son algunas reflexiones sobre los problemas que pueden tener las alternativas propuestas por dichos movimientos.

\section{Sobre los futuros}

1. La primera pregunta a hacerse es si este último movimiento es realmente mundial. Obvio que no lo es: las movilizaciones del 15-O no afectan a todos los países siendo clamorosa la ausencia africana y del sureste asiático, es decir, la de los países que Naciones Unidas llama ahora «menos desarrollados». Además, en los casos en los que sí afecta, no afecta a todos los ciudadanos, lo cual queda particularmente claro cuando las «manifestaciones masivas» son seguidas de triunfos electorales de los objetos del descontento. En el caso estadounidense, según encuesta de Gallup publicada a 21 de noviembre de 2011, el apoyo a «Occupy», que dice representar al «99\%», alcanzaría a un 25 por ciento de los encuestados, el rechazo a un 15 por ciento, pero la indiferencia o el desconocimiento llegaría al 60 por ciento ${ }^{4}$. Que los movilizados,

4. Los datos pueden verse en http://www.gallup.com/poll/150896/Support-OccupyUnchanged-Criticize-Approach.aspx?utm_source=alert\&utm_medium=email\&utm_ campaign=syndication $\& u t m \_$content $=$morelink $\& u t m \_t e r m=$ Politics $\% 20-\% 20$ USA 
aunque masivos, no sean mayoritarios es normal: algunos harán o se sentirán «vanguardia».

2. Pero lo anterior tiene consecuencias mayores para la siguiente pregunta, a saber, si estos movimientos proponen un cambio de sistema o un cambio en el sistema. El silencio o la relativa ausencia de planteamientos sobre países periféricos en los movimientos de los países centrales, sobre todo en los Estados Unidos, es tan audible como la relativa ausencia de movimientos acordes con aquellos en los países periféricos. Los movimientos locales contra un mal o conjunto de males que están a escala mundial tienen poco que hacer, a no ser que se crea en un «efecto mariposa», si no se coordinan a la escala en que se da el problema.

3. Para responder a esta pregunta, puede utilizarse la tipología de comportamientos que planteaba el sociólogo estadounidense Robert K. Merton a mitades del siglo pasado, aunque adaptándola a los presentes propósitos argumentales. Lo que aquél pretendía era hacer una tipología de las reacciones sociales ante situaciones de crisis, para lo cual usaba la actitud que se tomase hacia fines y medios socialmente aceptados, actitud que podía ser de aceptación (+) o de rechazo ( - ). A continuación se presentan los tipos de reacciones que provocan las crisis actuales de más frecuentes a menos frecuentes en particular en los países centrales.

\begin{tabular}{|l|l|l|}
\hline Tipo & Fines & Medios \\
\hline Conformista & + & + \\
\hline Ritualista & - & + \\
\hline Innovador & + & - \\
\hline Indignado & - & - \\
\hline Revolucionario & $+/-$ & + /- \\
\hline
\end{tabular}

Efectivamente, el conformismo es, con mucho, el comportamiento más frecuente: se observa en aquellos que aceptan tanto las metas del sistema (bienestar, felicidad, seguridad, identidad, libertad) como los medios legítimos para alcanzarlas (empleo, satisfacción, protección, representación política) pero, y esto es muy importante, con independencia de que los logren e incluso con independencia de que consigan acceder a los medios para alcanzarlos.

Después vendría el ritualismo de los que aplican mecánicamente los medios aceptados con independencia de que lleven al fin aceptado socialmente. Es propio de las burocracias, incluidas las burocracias políticas, pero también 
de determinadas formas de «pensamiento mágico» que pueden encontrarse en otras capas de la población.

Los innovadores son los que buscan nuevos medios para los fines aceptados: puede ser el delito de cuello blanco y la corrupción política o el pequeño delito callejero, aunque es obvio que, desde el punto de vista de los conformistas, es este último el que más preocupa, aunque el gran delito sea objetivamente más importante, pero no tan visible en la vida cotidiana de quien lee pocos periódicos de «información general». Pero también pueden ser (retóricas aparte) las nuevas formas de organización de sindicatos reformistas como medios para lograr los fines sancionados socialmente.

Los indignados forman el grupo del que se está hablando en el presente epígrafe: hay un rechazo de los fines y de los medios socialmente aceptados con el particular factor euforizante que produce la inmersión en un grupo de semejantes y que puede distorsionar la percepción de los problemas. Es preciso recordar los experimentos de psicología social de Muzafer Sherif y Solomon Asch sobre el peso que tiene el grupo en la percepción de estímulos ambiguos generando conformidad, pero también en conseguir errores de percepción incluso ante estímulos inequívocos. En todo caso, se impone una precaución a la hora de incluir a todos los participantes en el movimiento en la misma categoría, lo cual explicaría esa oscilación entre los que proponen cambios en el sistema y los que buscan un cambio de sistema.

En efecto, entre los conformistas y los ritualistas, si no alcanzan las metas sociales, puede producirse y, de hecho, se producen diferentes niveles de frustración dependiendo del tipo de personalidad, del contexto y de la profundidad de la frustración y del grado de miedo que los dirigentes políticos y sociales han conseguido imbuirles (Estefanía, 2011). Los frustrados pueden confundirse con los indignados. Ahora bien, a no ser que se ponga en funcionamiento un mecanismo de defensa de negación (negando el problema o, por lo menos, su gravedad y duración), la frustración genera agresividad y la agresividad busca un objeto sobre el que descargarse y puede hacerlo de forma violenta en cuyo caso no estaríamos en el tipo innovador sino en una variante de los dos primeros. Estos agresivos pueden dirigir su agresividad hacia sí mismos (y por eso se explica el aumento de suicidios en los países hasta ahora centrales) o hacia fuera, por ejemplo en forma del uso del «Otro» (inmigrante, de diferente religión o sexualidad o supuesta «raza») como objeto de la propia agresividad física o política como se ve en el auge de los partidos xenófobos en Europa comenzando por Austria y Suiza donde casi alcanzan a un tercio de los votantes. Si quieren algo, es un cambio en el sistema. 
4. El reto para los «indignados» es claro: cómo pasar de un comportamiento expresivo de la indignación (comportamiento emocional diferente al de la agresividad) a un comportamiento instrumental (racional con respecto a fines) en el que se pongan en práctica nuevos medios para alcanzar fines igualmente nuevos, que es lo que Merton calificaba como revolucionario. Fines probables y medios disponibles para que no se trate de un «wishful thinking», de una infantil y freudiana «omnipotencia de las ideas» y que no excluye que la acumulación de cambios locales en el sistema alcance una masa crítica y, de la cantidad a la calidad, pueda producir un cambio de sistema y no solo cambios en el sistema como pretenden algunos conformistas y ritualistas frustrados. Pero el cambio de sistema no se sabe hasta que se produce, lo cual no quita para tener en cuenta la posibilidad de falsas alternativas que van de lo puramente retórico al etnicismo (Amin, 2011) o las posibilidades de alianzas con otras fuerzas como las sindicales (Wallerstein, 2012). Las dudas, en el 15M, sobre su propia organización (seguir siendo horizontales y asamblearios, pasar a organización estable o incluso a partido) van en esa línea.

Porque una cosa es apuntar con el dedo, y certeramente, a qué está mal (Žižek, 2011) y otra cosa muy diferente es proponer vías viables de cambiarlo y no solo «propuestas» para ver si alguien las recoge, aunque no se excluye lo que Susan George, en su trabajo sobre la OMC, llamó el «efecto Drácula», a saber, el efecto que se produce cuando determinados asuntos se airean y se sacan a la luz del sol. Es, si se prefiere, el síndrome de «el rey está desnudo». Se trata, a fin de cuentas, de hacer problemática «esta situación de incompresible respeto por parte del público en general» que es «lo que necesita el financiero para que la democracia no le ate las manos» (texto del ya citado «El moderno Midas» de Bertrand Russell publicado en 1932). Si es cierto que «mayo del 68», en su complejidad y heterogeneidad, cambió por lo menos la percepción de muchas cosas, los movimientos actuales podrían hacer algo parecido aunque sea difícil pronosticar un resultado $u$ otro como se ha visto más arriba a propósito de piqueteros y forajidos.

Pero también es cierto que ahora es más fácil que las iniciativas locales tengan un impacto sobre la realidad mundial, dado el profundo desequilibrio en el que se encuentra el sistema mundial y, por tanto, la mayor probabilidad del «efecto mariposa», efecto producido por pequeños cambios en zonas marginales del sistema. También, probablemente, ahora es más necesario, dadas las crisis contemporáneas. 


\section{Bibliografía}

Acosta, A. (2005), La rebelión de los forajidos, La Insignia, abril, disponible en http://www.lainsignia.org/2005/abril/ibe_071.htm

Aguirre Rojas, C.A. (2012), Las revueltas populares de 2011 en perspectiva histórica, 26 de enero, disponible en http://es.scribd.com/doc/88324292/ Aguirre-Rojas-Carlos-Las-revueltas-populares-de-2011-en-perspectiva-historica- 2012

Álvarez-Ossorio, I. (2012), ¿Otoño islamista?, Próximo Oriente (blog), enero, disponible en http://proximooriente.blogspot.com/2012/01/otono-islamista. html

Amin, S. (2011), Audacia, más audacia, América Latina en movimiento, 13 de diciembre, disponible en http://alainet.org/active/51548

ARTAL, R.M. (2011), La energía liberada, Madrid: Aguilar.

BADRA, H. (2012), «The Israeli social protest movement», World Economic Association Newsletter, II, 1, 7, disponible en http://www.worldeconomicsassociation.org/Newsletter/Issue2-1.pdf

Chomsky, N. (2011), Occupy the future, In These Times, $1^{\circ}$ de noviembre, disponible en http://www.inthesetimes.com/article/12206/occupy_the_future/

ChOMsky, N. (2012), Occupy, Nueva York: Zuccotti Park Press.

Chouikha, L. (2011), Las nuevas tecnologías de la comunicación, vectores de la 'revolución democrática árabe': el caso tunecino, Awraq, 3, 97-106.

DOMĖNECH, X. (2011), Dues lògiques d'un moviment. Llegint el 15-M i els seus llibres, Hegemonies (blog), 22 de julio, diponible en http://inicis.blogspot. $\mathrm{com} /$

DoyLE, S. (2011), New Eden, old devils, In These Times, 22 de noviembre, disponible en http://www.inthesetimes.com/article/12275/new_eden_old_devils

EngelHardT, T. (2011), The United States of Fear, Chicago: Haymarket Books.

Estefanía, J. (2011), La economía del miedo, Madrid: Galaxia Gutemberg.

Fontana, J. (2011), Por el bien del Imperio. Una historia del mundo desde 1945, Barcelona: Pasado y Presente.

Gómez Vela, A. (2012), 17 claves para entender la marcha del Isiboro Sécure, en Horizontes nómadas (blog), abril, disponible en http://horizontesnomadas. blogspot.com.es/2012/04/17-claves-para-entender-la-marcha-en.html

GonZAlez-QuijAnO, Y. (2011), Túnez, El Cairo: la revolución árabe y sus orígenes digitales, Awraq, 3, 87-96.

Gorraiz LóPEZ, G. (2012), ¿Hacia la hambruna mundial?, ALAI - América Latina en movimiento, 5 de enero, disponible en http://alainet.org/active/51898

GutiérRez de TerÁN, I. y Álvarez-Ossorio, I. (2011), Informe sobre las revueltas árabes. Túnez, Egipto, Yemen, Bahrein, Libia y Siria, Madrid: Ediciones de Oriente y Mediterráneo. 
KeMPF, R. (2012), De Londres à Santiago, la révolte des déclassés, Le Monde diplomatique, mayo, págs. 24-25.

LEWIS, M. (2012), Boomerang. Viajes al nuevo Tercer Mundo europeo, Barcelona: Deusto.

Mason, P. (2012), Why It's Kicking Off Everywhere: The New Global Revolution, Londres: Verso.

Newman, A. (2012), Austerity and the End of the European Model, Foreign Affairs, $1^{\circ}$ de mayo, disponible en http://www.foreignaffairs.com/articles/137611/abraham-newman/austerity-and-the-end-of-the-europeanmodel?cid=nlc-this_week_on_foreignaffairs_co-050312-austerity_and_the_ end_of_the_e_2-050312

Prada Alcoreza, R. (2011), La guerra de la Madre Tierra, Horizontes nómadas (blog), diciembre, disponible en http://horizontesnomadas.blogspot. com/2011/12/la-guerra-de-la-madre-tierra-mas.html

Prada AlcorezA, R. (2012), Otra vez el TIPNIS, Horizontes nómadas (blog), abril, disponible en http://horizontesnomadas.blogspot.com.es/2012/04/otra-vezel-tipnis.html

REINHARDT C.M. y RogofF, K.S. (2011), Esta vez es distinto. Ocho siglos de necedad financiera, Madrid: Fondo de Cultura Económica.

Smith PH. B. y MAX-NeEF, M. (2011), Economics Unmasked, Totnes, Devon: Green Books.

Stiglitz, J. E. (2011), The globalization of protest, Project Syndicate, 11 de noviembre, disponible en http://www.project-syndicate.org/commentary/ stiglitz144/English

Stiglitz, J.E. (2012), The Book of Jobs, Vanity Fair, enero, disponible en http:// www.vanityfair.com/politics/2012/01/stiglitz-depression-201201

StREeCK, W. (2011), The crises of democratic capitalism, New Left Review, 71, Septiembre-Octubre, disponible en http://newleftreview. org/?page $=$ article $\&$ view $=2914$

STRYKER, D. (2011), "Why Iceland should be in the news, but is not», Daily Kos, $1^{\circ}$ de agosto, disponible en http://www.dailykos.com/ story/2011/08/01/1001662/-Icelands-On-going-Revolution

THERBORN, G. (2007), «The European present as World history», Alternativas. Cuadernos de Trabajo Social. 15, diciembre, pp. 17-28, disponible en http:// rua.ua.es/dspace/handle/10045/12859

Varios Autores (2011a), De indignaciones y alternativas, América Latina en Movimiento, vol. XXV, $\mathrm{n}^{\circ} 471$, monográfico. Disponible en http://alainet.org/publica/alai471w.pdf

Varios Autores (2011b), Les veus de les places, Barcelona: Icaria.

Varios Autores (2011c), La rebelión de los indignados: Movimiento 15 M: Democracia real, iya!, Madrid: Popular. 
VIVAS, E., (2012), De la economía verde, 1@s indignad@s y los foros sociales, América Latina en Movimiento, 30 de enero, disponible en http://alainet.org/ active/52407

Wallerstein, I. (2011), El fantástico éxito de Ocupa Wall Street, Commentaries, n. 315, 15 de octubre, disponible en http://www2.binghamton.edu/fbc/commentaries/archive-2011/315spj.htm

Wallerstein, I. (2012), May Day: The Return of the Trade-Unions?, Commentaries, n. 328, $1^{\circ}$ de mayo, disponible en http://www.binghamton.edu/fbc/ commentaries/

Williamson, J.G. (2012), Comercio y pobreza. Cuándo y cómo comenzó el atraso del Tercer Mundo, Barcelona, Crítica.

ŽIŽEK, S. (2011), The violent silence of a new beginning, In These Times, 26 de octubre, disponible en http://www.inthesetimes.com/article/12188/ the_violent_silence_of_a_new_beginning/ 